\title{
Topiramate Induced Aqueous Misdirection Syndrome Presenting as Bilateral Elevated Intraocular Pressure-A Case Report
}

\author{
Didar Abdulla $^{1}$, Ojasvi Sharma ${ }^{2}$ and Tarun Sharma ${ }^{3 *}$ \\ ${ }^{1}$ Clinical Research fellow in Ophthalmology, Royal Surrey Hospital, Guildford, United Kingdom \\ ${ }^{2}$ Medical student, University of Nottingham, UK \\ ${ }^{3}$ Consultant Ophthalmic surgeon, Worcestershire Royal Hospitals NHS trust, UK
}

*Corresponding author: Tarun Sharma, Consultant Ophthalmic surgeon, Worcestershire Royal Hospitals NHS trust, UK.

Received Date: September 02, 2020

Published Date: October 23, 2020

\begin{abstract}
Aim: To describe a case of raised intraocular pressure due to Topiramate, to raise awareness of the condition, encourage patient education of side effects land reduce future misdiagnosis.

Method: A case report of a Topiramate induced aqueous misdirection and literature review in the English language.

Conclusion: Topiramate induced angle closure is an ophthalmic emergency that can potentially result in permanent vision loss if not promptly recognized and appropriately treated. It is a rare but serious adverse reaction in which angle closure occurs due to development of ciliochoroidal effusions. The early recognition and discontinuation of the offending medication is the most essential treatment of topiramate-induced angle closure. Patient education is a key prophylactic measure.
\end{abstract}

Key Words: Topiramate, Raised Intraocular Pressure, Aqueous Misdirection, Angle Closure Glaucoma

\section{Introduction}

Topiramate (brand name Topamax) is a commonly prescribed oral sulphamate medication. It is usually prescribed for prophylaxis against migraine and epileptic seizures. Few practitioners have started using it off-label as an effective treatment for various disorders, including neuropathic pain, binge eating disorder, alcohol use disorder, bulimia nervosa, obesity, borderline personality disorder, and idiopathic intracranial hypertension [1-3].

This case highlights that misdiagnosis is easily possible in the ophthalmology emergency rooms thus causing delay in correct treatment which may have adverse impact on the wellbeing of the patient. It is important for physicians and patients to recognize the medication's potential systemic and ophthalmic adverse effects.

\section{Cases Report}

A 46 year old Caucasian female initially presented to the Eye casualty department complaining of bilateral reduced vision and ocular discomfort, and was found to have elevated intraocular pressure of $35 \mathrm{mmHg}$ in her right eye and 33mmHg in her left eye. She had recently commenced treatment with Topiramate for long standing migraines, and was thought to have developed elevated intraocular pressure secondary to this. She was treated for acute angle closure glaucoma and followed up on a number of occasions in the Eye casualty department. Whilst the intraocular pressure was controlled, she was subsequently noted to have low grade bilateral anterior chamber inflammation. This was assumed to be a separate 
diagnosis. Throughout these initial visits, she felt that she was not given an adequate explanation for the cause of her symptoms or the likely prognosis of this condition. As she was not satisfied with the information she was given through the eye casualty department, she sought a second opinion. Fortunately, the consultant was able to unite all the clinical findings and explain that she had experienced a change in the configuration of the ciliary body following starting Topiramate (which is a known side effect of Topiramate treatment), causing aqueous misdirection, and secondary elevated intraocular pressure. She had subsequently developed a low grade anterior chamber inflammatory response following this. The consultant was able to perform gonioscopy to confirm that the anterior chamber drain age angle was open through 360 degrees in both eyes, and was therefore happy to dilate her to break a posterior synechiae which had formed in the right eye, and treated her with a reducing course of topical Prednisolone 1\% eye drops and Cosopt eye drops to control her intraocular pressure. She was seen once as follow-up by the consultant, at which point the episode had resolved and she was advised to taper all treatment to a stop.

The patient had no prior ocular history of inflammatory eye disease, a known history of migraines but reports no systemic inflammatory disease. Review of systems is otherwise unremarkable.

It took her 3 months to return to normal. Her vision improved to $6 / 4$ in each eye with normal intraocular pressure at $17 \mathrm{mmHg}$ in the righteye and $15 \mathrm{mmHg}$ in the left eye. Optic discs were healthy with cup to disc ratio of 0.2 in both eyes. There was no active anterior chamber or posterior segment inflammation in either eye. As an adjunct, she reported intermittent episodes of discomfort affecting the right eye, which was described as a gritty foreign body sensation and occasional film across her vision. On examination it was noted that she had a degree of low grade ocular surface inflammation which may have been caused by her recent intensive topical treatment. Since stopping Topiramate 12 months ago, there have been no recurrence of symptoms. The patient remains symptom free on topical lubricant treatment with The aloz Duo preservative-free lubricant drops as required to both eyes.

\section{Discussion}

Three per 100,000 patients taking topiramate may develop Topiramate-induced angle closure glaucoma. 4 Its incidence is likely to increase as indications for the drug expand. This condition has been reported to occur more commonly in female patients, because the medication is more commonly used by women [4]. Topiramate-induced angle closure is an ophthalmic emergency that can potentially result in permanent vision loss if not promptly recognized and appropriately treated. It is a rare but serious adverse reaction in which angle closure occurs due to development of ciliochoroidal effusions. This results in anterior rotation of the ciliary body and forward displacement of the lens-iris diaphragm [5]. The precise mechanism of angle closure is not fully understood. Topiramate is thought to cause accumulation of fluid within the choroid and ciliary body due to an increase in permeability of the vasculature. The thickening of the choroid results in increased retro lenticular pressure, which pushes the iris-lens diaphragm, forward [6]. Topiramate-induced angle closure occurs in the majority of cases within the first two weeks after initiation of treatment or increase in dose 6 , although it may develop after many months of taking the medication [7].

Patients presenting with raised intraocular pressure and angle closure glaucoma should be carefully assessed as the management for primary angle closure glaucoma is of little benefit in secondary angle closure glaucoma induced by Topiramate. Sulpha drugs such as acetazolamide are a cause of ciliary rotation and should be avoided in topiramate induced angle closure. The early recognition and discontinuation of the offending medication is the most essential treatment of topiramate-induced angle closure. Patient education is a key prophylactic measure. They should be educated on the symptoms of angle closure and encouraged to see an ophthalmic professional urgently if they experience such symptoms.

Ophthalmic professional should inform the physician who prescribed it so that they can use an alternative medication for the management of the condition for which topiramate was prescribed. Ophthalmic management includes a cycloplegic agent such as atropine or cyclopentolate, and anti-inflammatory agent along with topical glaucoma medications. Laser peripheral iridotomy (LPI) is unlikely to resolve the episode as pupillary block is not the mechanism and may exacerbate the inflammation. The glaucoma drainage procedures are recommended for this as a first-line therapy [8].

\section{Acknowledgement}

None.

\section{Conflict of Interest}

Author declares no conflict interest.

\section{References}

1. Shen WW (2018) Anticraving therapy for alcohol use disorder: A clinical review. Neuropsychopharmacol Rep 38(3): 105-116.

2. Grootens KP, Meijer A, Hartong EG, Doornbos B, Bakker PR, et al. (2018) Weight changes associated with antiepileptic mood stabilizers in the treatment of bipolar disorder. Eur J Clin Pharmacol 74(11): 1485-1489.

3. Celebisoy N, Gokcay F, Sirin H, Akyurekli O (2007) Treatment of idiopathic intracranial hypertension: topiramate vs acetazolamide, an open-label study. Acta Neurol Scand 116(5): 322-327.

4. Abtahi MA, Abtahi SH, Fazel F, Roomizadeh P, Etemadifar M, et al. (2012) Topiramate and the vision: a systematic review. Clin Ophthalmol 6: 117131.

5. Lan YW, Hsieh JW (2017) Bilateral acute angle closure glaucoma and myopic shift by topiramate-induced ciliochoroidal effusion: case report and literature review. Int Ophthalmol 38(6): 2639-2648.

6. Fraunfelder FW, Fraunfelder FT, Keates EU (2004) Topiramate-associated acute, bilateral, secondary angle-closure glaucoma. Ophthalmology 111(1): 109-111.

7. Czyz CN, Clark CM, Justice JD, Pokabla MJ, Weber PA (2014) Delayed topiramate-induced bilateral angle-closure glaucoma. J Glaucoma 23(8): 577-578.

8. Parikh R, Parikh S, Das S, Thomas R (2007) Choroidal drainage in the management of acute angle closure after topiramate toxicity. J Glaucoma 16(8): 691-693. 